\title{
Optical pulse differentiation based on a resonant slow \& fast light system
}

\author{
Sanghoon Chin ${ }^{1}$, Tae-Jung Ahn² and Luc Thévenaz ${ }^{1}$ \\ ${ }^{1}$ Ecole Polytechnique Fédérale de Lausanne, STI-GR-SCI-LT, Station 11, CH-1015 Lausanne, Switzerland \\ ${ }^{2}$ Department of Photonic Engineering, Chosun University, 375 Seosuk-dong, Gwangju, South Korea \\ sanghoon.chin@epfl.ch
}

\begin{abstract}
We experimentally demonstrate that temporal differentiation of optical pulses can be realized in a slow \& fast light system based on a resonance. The waveform of a 13 ns Gaussian pulse was experimentally first-order differentiated.

OCIS codes: (060.4370) Nonlinear optics, fibers; (290.5900) Scattering, stimulated Brillouin; (070.6020) Signal processing
\end{abstract}

Development of basic mathematical operations in all-optical signal processing circuits - such as differentiators, integrators and logic gates - is attracting an increasing interest in the optical community, since they are key functions required for all-optical computing and networks. All-optical temporal differentiators and integrators have been experimentally realized in optical fibers, based on a single uniform long-period fiber grating (LPG) and a phaseshifted fiber Bragg grating. In this paper, we propose a new technique that can provide a first-order derivative of an arbitrary optical waveform, using a resonance-based slow \& fast light system.

Sharp spectral resonances can generate a strong dispersion leading to a substantial change of the effective group velocity at the center of the resonance. Neglecting the higher order dispersion terms, the spectral distribution of the pulse exiting from such a system $E_{\text {out }}(\omega)$ is well approximated by [1]:

$$
E_{\text {out }}(\omega) \approx \exp \left(\frac{G}{2}\right) \exp \left[-i \frac{G}{\Gamma_{B}}\left(\omega-\omega_{o}\right)\right] E_{\text {in }}(\omega),
$$

where $e^{G}$ is the net signal power gain, $\omega_{0}$ is the central frequency of the resonance, $\Gamma_{B}$ is the resonance FWHM width and $E_{i n}(\omega)$ is the pulse input spectrum. According to the Fourier transform properties, it can be simply anticipated that the output pulse will be temporal shifted by an amount of $G / \Gamma_{B}$ and the effective delay can be tuned by varying $\mathrm{G}$, which is essentially the principle of resonance-based slow \& fast light. For small frequency detunings $\omega-\omega_{0}$, the phase shifts satisfies the condition $G / \Gamma_{B}\left(\omega-\omega_{0}\right) \ll 1$ and the transfer function can be further simplified as:

$$
E_{\text {out }}(\omega) \approx \exp \left(\frac{G}{2}\right)\left[1-i \frac{G}{\Gamma_{B}}\left(\omega-\omega_{o}\right)\right] E_{\text {in }}(\omega) .
$$

It is interesting to point out that the second term in the transfer function is the required spectral response for a firstorder differentiator and the output signal in the time domain can be simply expressed by this sum.

$$
E_{\text {out }}(t) \approx \exp \left(\frac{G}{2}\right)\left[E_{\text {in }}(t)-\frac{G}{\Gamma_{B}} E_{\text {in }}^{\prime}(t)\right]
$$

The output signal is formed by the sum of the input signal plus its derivative weighted by the gain factor. For large gains the derivative term can be strongly dominant and leads to a simple visual interpretation of the delay/advancement effect and of the signal temporal shapes, as illustrated by the experimental traces shown in Fig. 1 for a fast light system. The sign of the derivative is simply reversed for a slow light system.

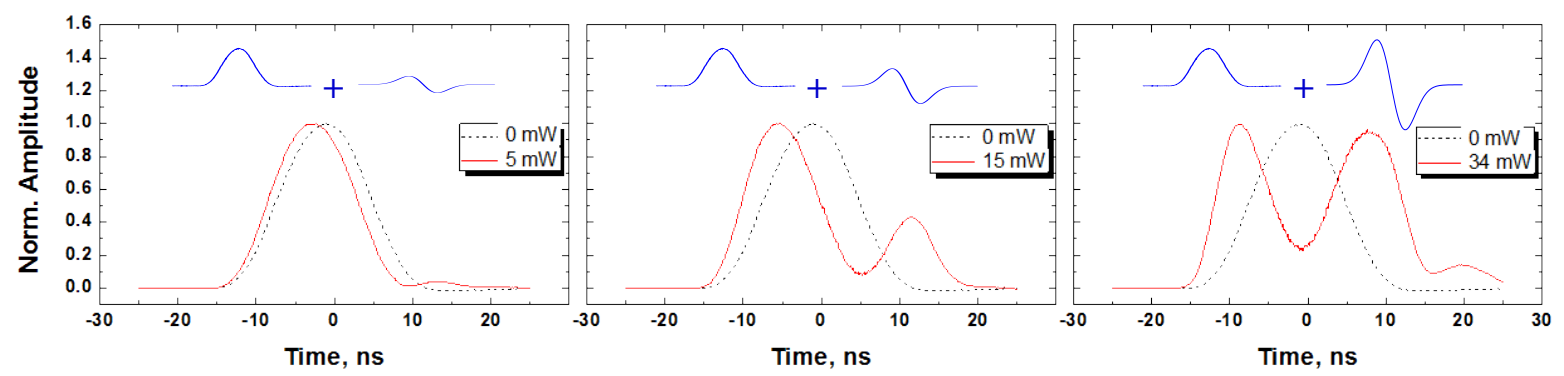

Fig. 1. Time traces of an optical pulse exiting from a Brillouin fast light system for different pump powers (black line represents the input pulse). The insert depicts the sum given by Equ.3 to interpret the experimental traces squared (thus rectified) by the power-dependent photodetection.

\section{References}

[1] Z. Zhu, D. J. Gauthier, Y. Okawachi, J. E. Sharping, A. L. Gaeta, R. W. Boyd, and A.E. Willner, "Numerical study of all-optical slow-light delays via stimulated Brillouin scattering in an optical fiber," J. Opt. Soc. Am. B 22, 2378-2384 (2005). 